\author{
Associate Professor Daniel PEPTENATU,PhD \\ E-mail:daniel.peptenatu@geo.unibuc.ro \\ Ion ANDRONACHE,PhD \\ E-mail:ion.andronache@geo.unibuc.ro \\ Andreea Karina GRUIA,PhD Student \\ E-mail:karina.gruia@yahoo.com \\ Alexandra GRECU,PhD Student \\ University of Bucharest \\ E-mail: alexandragrecu28@gmail.com \\ Assistant Professor Cristina DIMA,PhD \\ E-mail:cristina.dima@man.ase.ro \\ Professor Răzvan Cătălin DOBREA,PhD \\ E-mail:razvan.dobrea@man.ase.ro \\ Associate Professor Dumitru Alexandru BODISLAV, PhD \\ E-mail:dumitru.bodislav@economie.ase.ro \\ The Bucharest University of Economic Studies
}

\title{
APPLICATION OF FRACTAL-STRUCTURAL METHODS IN THE ANALYSIS OF SPATIAL DISTRIBUTION OF THE TURNOVER IN ROMANIA
}

\begin{abstract}
The study proposes a fractal-textural qualitative analysis method of disparities in the turnover in Romania, comprising GLCM entropy, fractal entropy and Higuchi $2 D$ dimension. In the open source QuantumGIS platform there were developed cartographic models of the turnover distribution in Romania, at the level of territorial administrative units, for each year between 2000 and 2016. For each year there were established five levels for representing the territorial administrative units, for which a structural analysis of the levels was conducted: 1+2-the smallest values, 3- the mean values and 4+5-the highest values. The proposed model highlighted numerous specificities in the turnover distribution in each development region, the most important being the modeling of the structural crisis in 2009. Perhaps the most important aspect highlighted is the importance of level 3 in generating critical masses for levels $1+2$ and $4+5$.
\end{abstract}

Keywords: Fractal analysis, Fractal entropy, Higuchi 2D, Turnover.

JEL Classification: C02, C15, C55, P25

DOI: $10.24818 / 18423264 / 54.1 .20 .04$ 
Daniel Peptenatu, Ion Andronache, Andreea Karina Gruia, Alexandra Grecu, Cristina Dima, Răzvan Cătălin Dobrea, Dumitru Alexandru Bodislav

\section{Introduction}

The structural dynamics of economic processes is one of the directions that provide valuable information in understanding the regional manifestation patterns. The spatial dynamics of the development and the trends in territorial concentration increasingly concern the scientific world as well as the decision makers. Despite this interest, the number of studies is relatively low, and they are targeting punctual, generally quantitative approaches (Allen and Arkolakis, 2014; Harrison et al. 2011).

In the polycentric development studies, it is analyzed the manner whereby the economic activities are concentrated along the development axes connecting the polycentric nuclei (Boix and Trullén,2007, Meijers et al., 2007, Geppert and Stephan, 2008, Wang and Kockelman, 2009).

In numerous studies regarding the economic pressure on forestry resources, fractal and nonfractal models were applied to identify patterns in the manifesting of this phenomenon, with very high disparities in former communist countries (Andronache et al, 2017; Pintilii et al. 2017). The obtained results may be easily extrapolated for the analysis of the regional disparities in the turnover distribution.

The results obtained in the risk analysis based on entropy can contribute to the development of methodologies for analyzing the spatial dimension of the occurrence of economic phenomena (Jiang et al., 2009; Parker, 2017). Also, the proposed method can make an important contribution to the development of methodologies for the analysis of the impact of economic restructuring on emerging processes occurring in a region.

\section{Data and Methodology}

\subsection{Spatialization of the turnover}

In order to perform such structural analysis a set of 15 anisotropic digital images was created, using QuantumGIS, as 2D representations of the territorial dynamics in turnover, for the period 2000-2016.

For each year, five representative ranges were established, for which a structural analysis of 1+2 - smallest values, 3-average values and 4+5-highest values was performed, for all the development regions of Romania (Figure 1). Considering that the Bucharest-Ilfov Development Region is surrounded by South Muntenia Development Region, fractal and non-fractal analysis of the latter would have been impossible, so the two were analyzed together. 
Application of Fractal-structural Methods in the Analysis of the Spatial Distribution of Turnover in Romania

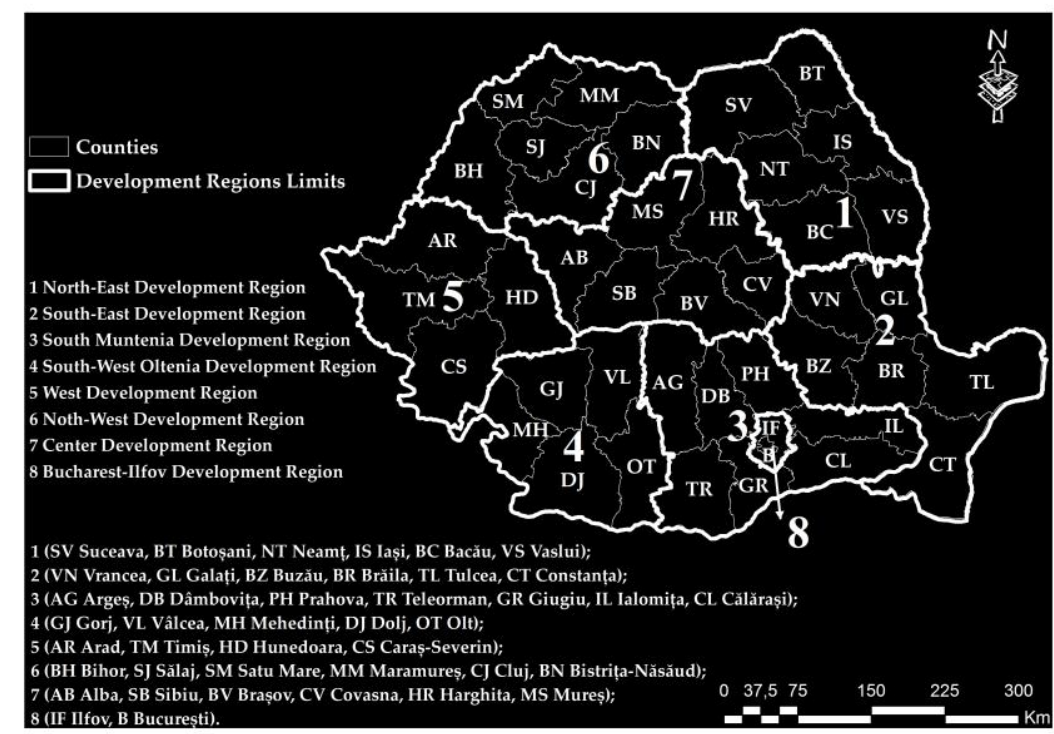

Figure 1.Structural dimension of the study area

\subsection{Image pre-processing}

The images obtained from QuantumGIS in order to determine GLCM Entropy, fractal entropy and Higuchi 2D were converted to 8-bit gray scale images using Image J 1.52o (Schneider et al. 2012). For the Fractal entropy analysis, the 8bits imaging in gray scale were segmented and then converted to binary using the same Image J 1.52o software. The pixels of interest fractals (foreground) were extracted in white, and the background pixels, non-fractals (background) were extracted in black. Through segmenting and binarizing, two sets of images were obtained: some with reduced turnover values (ATUs (administrative territorial units) poor and very poor - level $1+2$ ). And another set with high turnover values (rich and very rich ATUs - level $4+5$ ). Please note that level 3 represents moderate turnover on ATU level.

\subsection{Analysis of images}

For the qualitative analysis of the disparities in the turnover spread, GLCMentropy, fractal entropy and Higuchi dimension 2D were determined.

GLCM Entropy is a component of GLCM (Gray-Level Co-Occurrence Matrix). GLCM is a statistical method for examining the texture of an image in grayscale. Therefore, it no longer requires binarization, the method wherein, in most cases, quantitative and qualitative elements are lost. GLCM provides information on the spatial relationships of pixels in an image in grayscale, creating an array and then extracting statistical measures from this array (Haralick et al.

DOI: 10.24818/18423264/54.1.20.04 
Daniel Peptenatu, Ion Andronache, Andreea Karina Gruia, Alexandra Grecu, Cristina Dima, Răzvan Cătălin Dobrea, Dumitru Alexandru Bodislav

1973). In GLCM, entropy is a statistical measure of randomness and measures the degree of clutter or complexity of an image. Entropy is significant when the image is not consistent from a texture point of view and when many GLCM elements have low values. Complex textures tend to have high entropy.

Entropy is measured according to the equation (Haralick et al. 1973):

$$
\text { Entropy }=\sum_{i} \sum_{j} p(i, j) \log p(i, j)
$$

where $p(i, j)$ are the spatial co-occurrence probabilities,

where $i$ and $j$ are the coordinates of the co-occurrence matrix.

The GLCM entropy analysis was conducted using the Texture Analyzer plugin (Cabrera 2005) for Image J 1.52o software (Schneider et al. 2012).

Fractal entropy or information dimension $\left(D_{l}\right)$ is a measure of the amount of "disorder" present in an image. To evaluate $D_{l}$, probabilities are calculated to find points in the image (Pitsianis et al., 1989). Fractal entropy is derived from the Box-Counting analysis. If Box-Counting considers a box as the foreground part of the fractal objects whether it contains one point or a relatively high number of points. $D_{l}$ comes with a different approach and actually allocates weights to the boxes so that the boxes containing a higher number of points weigh more than boxes with fewer points. Fractal entropy is calculated only for binary images, as a needed prerequisite it is necessary to extract the fractal objects to be analyzed by binarization.

$D_{l}$ is defined by the following equation:

$$
\begin{aligned}
& D_{1}=\lim _{\varepsilon \rightarrow 0}\left[\frac{S(r+\varepsilon)-s(r)}{\log (r+\varepsilon)-\log (r)}\right], \text { and } \\
& \qquad S(r)=\lim _{N \rightarrow \infty} \sum_{i=1}^{N(r)} m_{i} \log \left(m_{i}\right)
\end{aligned}
$$

where $S(r)$ is Kolmogorov entropy, $\mathrm{N}$ is the number of boxes and $m_{i=} \frac{M_{i}}{M}, M_{i}$ is the number of points in $i^{\text {th }}$ box, $M$ is the total number of dots of the fractal object, and $\varepsilon$ is the size of the box

$D_{l}$ is calculated starting from the gradient of the entropy line diagram Kolmogorov of logarithms on the sides of the boxes used to cover the fractal object (Kunicki et.al. 2009). Like box-counting, the value $D_{l}$ is independent of the basis of the logarithm used.

Higuchi dimension 2D (H2D)is a fractal analysis of image texture, using grayscale images, as in the case of GLCM entropy. $H 2 D$ is a generalization of $H 1 D$ to calculate the fractal dimension of a plane curve (Spasic 2014). $H 2 D$ is based on the calculation of the fractal complexity of an image, considering the relationship between the gray levels of some pixels and the gray levels of the pixels in their proximity. TheH2D dimension is obtained from the negative trend of double logarithmic plot of values $\mathrm{A}(\mathrm{k})$ and $\mathrm{k}$ : 
Application of Fractal-structural Methods in the Analysis of the Spatial Distribution of Turnover in Romania

$$
D^{\text {KfoldDiff }}=-D
$$

where, $D$ is obtained from the relation $A(k) \propto k^{D}$ where $A(k)$ :

$$
A(k)=\frac{1}{k^{2}} \sum_{n=1}^{k} \sum_{m=1}^{k} A_{n, m}(k)
$$

And $A n, m(k)=$

$$
\begin{aligned}
A_{n, m}(k) & =\frac{1}{k^{2}}\left\{\left(\sum _ { i = 1 } ^ { \omega _ { n } ^ { k } } \sum _ { j = 1 } ^ { \omega _ { m } ^ { k } } \left[\left|x_{i-1, j}^{k}-x_{i-1, j-1}^{k}\right| k+\left|x_{i, j}^{k}-x_{i-1, j}^{k}\right| k\right.\right.\right. \\
& \left.\left.\left.+\left|x_{i, j-1}^{k}-x_{i-1, j-1}^{k}\right| k+\left|x_{i, j}^{k}-x_{i, j-1}^{k}\right| k\right] \frac{1}{4}\right) \Omega_{n, m}^{k}\right\}
\end{aligned}
$$

where $n$ and $m$ are the starting points that are heading towards $k$;

$i$ and $j$ are indices of individual points;

$\omega_{n}^{k}$ and $\Omega_{n, m}^{k}$ are normalization factors.

$k$ is calculated by multiplying the difference in gray levels of two adjacent pixels by the distance between the two pixels; again, $x$ is the pixel analyzed (Ahammer et al., 2015).

Higuchi 2D analysis was done automatically using the open-source software IQM 3.5.0(Kainz et al. 2015). After the preliminary tests, we opted for a maximum $\mathrm{k}=$ 1,137 considering that all ATUs would have a level 5 turnover $D^{K \text { foldDiff }}=$ 2.3051 , and if they had a level 1 turnover $D^{\text {KfoldDiff }}=2.8981$. It means that the more the influence of the turnover of the polarization centers on the nearby ATU in the neighborhood increases, $D^{\text {KfoldDiff }}$ will decrease.

\section{Results}

\subsection{Dynamics of turnover}

After 2000, Romania recorded a significant economic growth as a result of the structural reforms determined by the perspective of integration in the European Union. The turnover recorded an important increase during the whole analyzed period, interrupted only by the economic crisis that had an effect starting with 2009.

The analysis of the turnover dynamics at national level highlights the highest values in urban systems with a population of over 250000 inhabitants (Bucharest, Brasov, Cluj-Napoca, Timişoara, Constanta, Iaşi and Craiova), where the level 5 was maintained over the whole period, while level 4 is dominant in their emerging territorial system. By the end of the analyzed period, two emerging territorial systems, Bucharest and Brasov, recorded significant increases, with level 5 dominating (Figure 2). The levels1+2 is reduced towards the end of the period, when it still occupies compact geographic areas only in the South-West

DOI: 10.24818/18423264/54.1.20.04 
Daniel Peptenatu, Ion Andronache, Andreea Karina Gruia, Alexandra Grecu, Cristina Dima, Răzvan Cătălin Dobrea, Dumitru Alexandru Bodislav

development regions Oltenia and North East Region, two of the poorest regions of the European Union. In Figure 2, the turnover dynamics show the important role of the big urban centers in promoting territorial development.

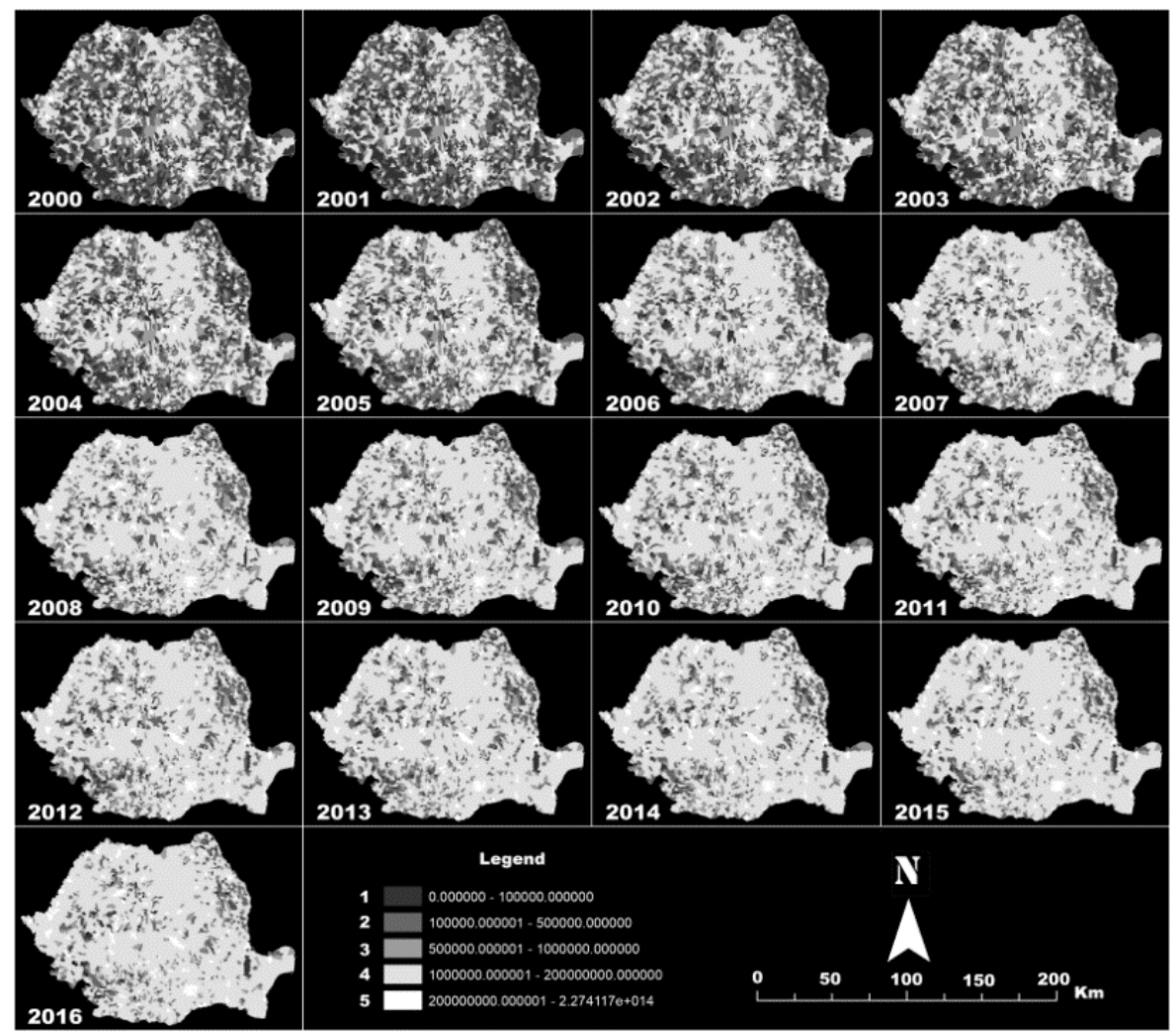

Figure 2.Turnover spread in Romania

In Figure $3 \mathrm{a}$, the evolution of Higuchi $2 \mathrm{D}$ size shows the stages in the evolution of turnover in Romania. Thus, simultaneously with the expansion of the areas with an increase in turnover, H2D dimension decreases. The application of fractal entropy (figure 3b) shows a higher fidelity in modeling the turnover dynamics in Romania, particularly after 2010.

The structural analysis through fractal entropy (figure 3c) highlights the particularities of the evolution of the three intervals: levels $1+2$, level 3 and levels $4+5$. Levels $1+2$ are continuously reduced over the period analyzed, towards the end of the interval being mostly concentrated in the South-West Oltenia and NorthEast development regions, where a trend towards compaction of the areas with low turnover values is noticed. Levels $4+5$ shows a significant increase fueled by level3, 
Application of Fractal-structural Methods in the Analysis of the Spatial Distribution of Turnover in Romania

but especially by $1+2$, which near large urban systems makes a quick pass to levels $4+5$.

In figures $4 \mathrm{a}, \mathrm{b}$ and $\mathrm{c}$ it may be seen the contribution of the entropy of each level to the GLCM entropy. Entropy levels 1+2 and GLCM entropy decrease over the whole analyzed period amid the shrinking and compaction of geographical areas in this interval (Figure 4a). The correlation between GLCM entropy and level 3 is reduced (figure 4a), the average level being characterized by a very high spatial disorder, being the interval towards which the units of the geographical spaces in the lower level and supplier for the upper level tend to establish. The correlation of entropy GLCM with entropy of the upper level is very good, the compaction of geographical areas with high turnover values being obvious (Figure $4 c)$.

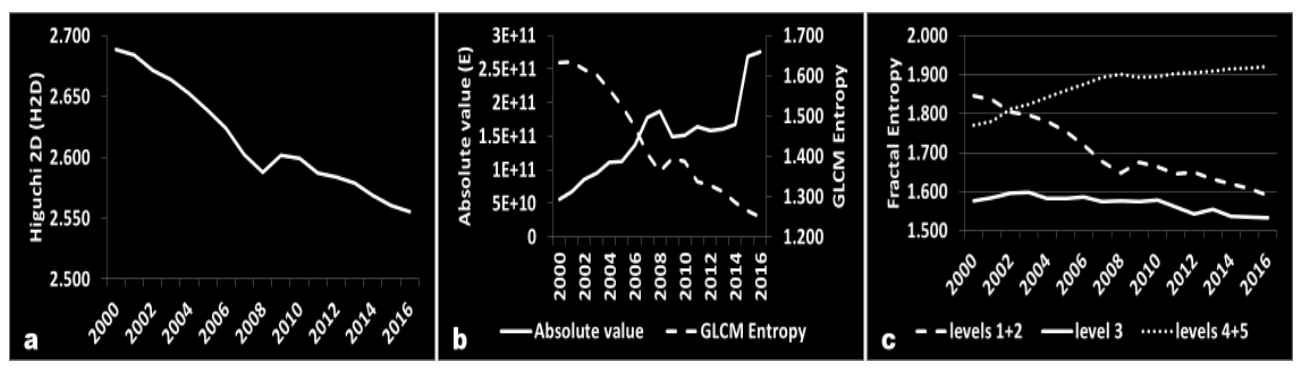

Figure 3 a.-H2D dynamics of turnover; b.-dynamics of fractal entropy and absolute value of turnover; c. - structural dynamics of fractal entropy of levels $1+2,3$ and $4+5$ of turnover at national level

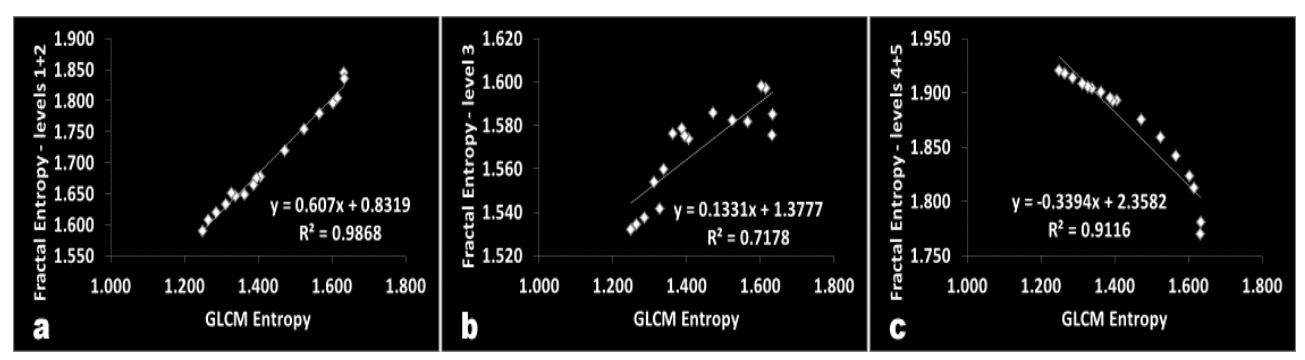

Figure 4. GLCM and fractal entropy dynamics-Romania: a.-correlation between GLCM entropy and the fractal entropy of the low values level (levels $1+2$ ); b.- correlation of GLCM entropy and fractal entropy of medium values-level 3 areas; c.- correlation of GLCM entropy and the fractal entropy of high values (levels 4+5) areas.

Figure 5 shows the entropy dynamics in the poorest development region in Romania and one of the poorest in the European Union, the North-East Development Region. The application of the H2D algorithm (figure 5a) and the

DOI: $10.24818 / 18423264 / 54.1 .20 .04$ 
Daniel Peptenatu, Ion Andronache, Andreea Karina Gruia, Alexandra Grecu, Cristina Dima, Răzvan Cătălin Dobrea, Dumitru Alexandru Bodislav

GLCM entropy (figure 5b) displays a decreasing tendency for the whole analyzed period except for 2009, when the effects of the economic crisis determined an entropy increase for two years. The structural analysis reveals a series of evolution specificities, thus levels $1+2$ shows a general decrease of entropy, but lower than other regions (figure 5c). The correlation between GLCM entropy and fractal entropy of each levels highlights the peculiarity of this region, the dependence of the general entropy on the entropy of levels $1+2$ (figure 5d) with the largest spread over the whole period. The reduced correlation between GLCM entropy and level3 (Figure 5e) shows the very low share of administrative-territorial units in this level and the very low dynamics. The correlation of GLCM entropy with the level with the highest turnover values (Figure 5f) registered an entropy increase against the increases recorded around the main cities in the region.

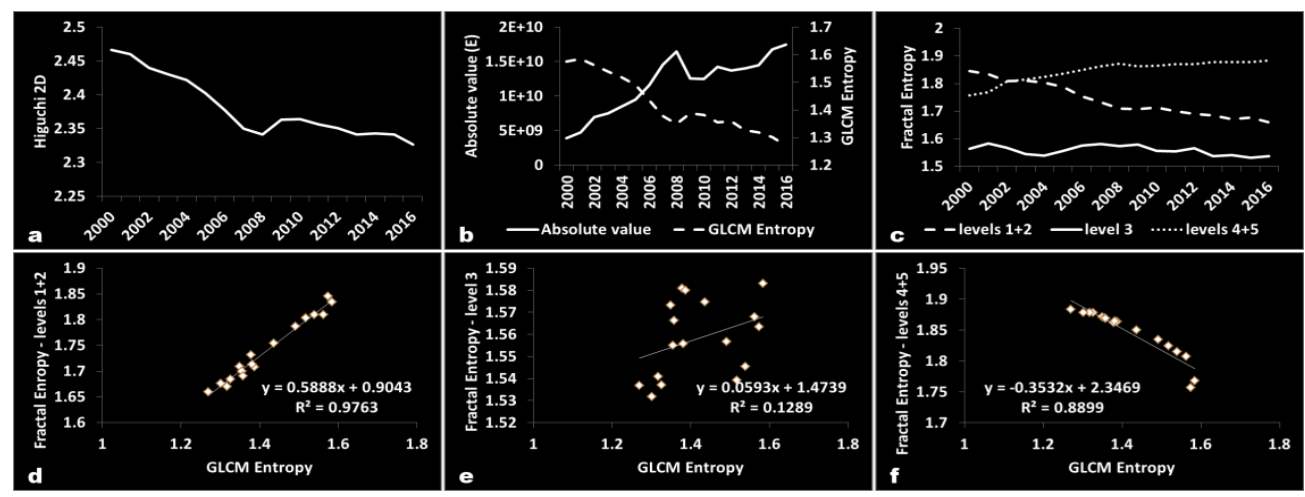

Figure 5. GLCM and fractal entropy dynamics-North-East

Development Region: a.- H2D dynamics; b.-GLCM entropy dynamics; c.-structural dynamics of fractal entropy; d.-correlation between GLCM entropy and the fractal entropy of low values levels $(1+2)$; e.- correlation GLCM entropy and the fractal entropy of medium values-level 3; f.- correlation of GLCM entropy and the fractal entropy high value levels (4+5).

The H2D and GLCM entropy analysis for the South-East Development Region highlights the decrease of entropy and a high amplitude of dynamics, showing a compaction of levels $4+5$ towards the end of the analyzed period (figure $6 \mathrm{a}$, figure $6 \mathrm{~b}$ ). At structural level, the amplitude of the decrease of entropy for the levels $1+2$ and $4+5$ (figure $6 \mathrm{c}$ ) is noted, which at the end of the analyzed period is reduced to several geographically isolated territorial administrative units. The correlation between GLCM entropy and fractal entropy of each levels shows the very good correlation between the levels $1+2$ and $4+5$ and the GLCM entropy (Figure 6d, Figure 6f), the two levels being dominant at the beginning, respectively 
Application of Fractal-structural Methods in the Analysis of the Spatial Distribution of Turnover in Romania

at the end of the period. It is noticed the correlation between GLCM entropy and level $3, \mathrm{R}^{2}$ recording only 0.6533 (Figure 6e).

Figure 7 analyzes the development regions Sud-Muntenia and BucharestIlfov, the geographic space with the greatest economic complexity. Entropy of GLCMand H2D records the highest amplitude of the entropy evolution (Figure 7a, Figure $7 b)$. Fractal entropy of $1+2$ levels recorded the highest decrease among all analyzed regions (Figure 7c), such evolution showing the capital city's economic force over an ever-expanding space with values in the levels4+5. The fractal entropy of the $4+5$ levels recorded a continuous increase, being the level that extends almost to the entire area under review. Entropy of level 3 shows a continuous reduction trend by narrowing this level in favor of levels $4+5$. The influence of fractal entropy of each levels on GLCM entropy is highlighted by the good correlation with the levels $1+2$ (Figure $7 d$ ) and $4+5$ (Figure 7f), and by the reduced correlation with the level 3 (Figure 7e).

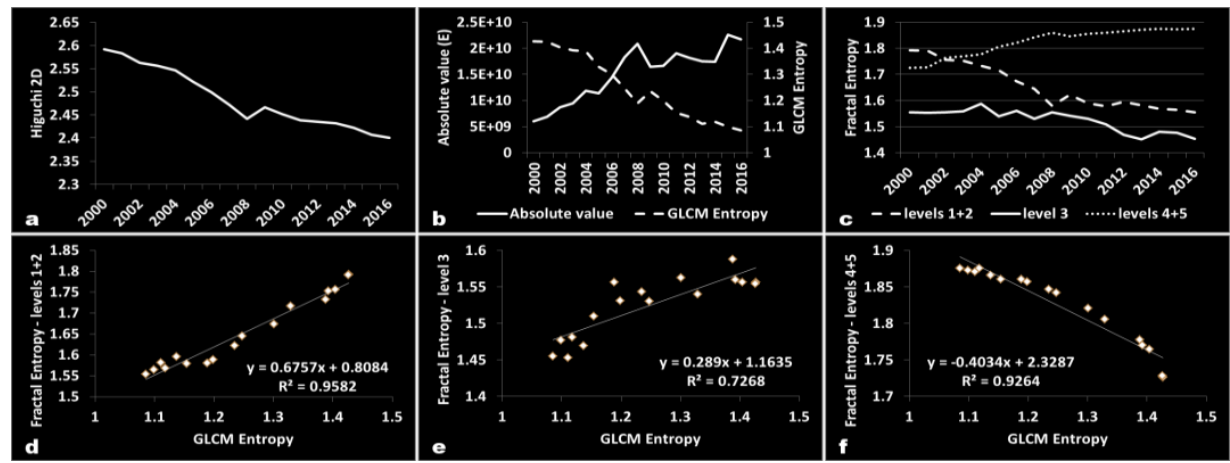

Figure 6. GLCM and fractal entropy dynamics-South-East Development Region: a.- H2D dynamics; b.-GLCM entropy dynamics; c.-structural dynamics of fractal entropy; d.-correlation between GLCM entropy and the fractal entropy of low values levels $(1+2)$; e.- correlation GLCM entropy and the fractal entropy of medium values-level 3; f.- correlation of GLCM entropy and the fractal entropy high value levels (4+5).

At the level of South Development Region (Muntenia), the GLCM and H2D analysis shows the greatest dynamics. The polarizing role of the capital city's footprint marks the entropy evolution in the entire region through the highest decrease in the H2D algorithm (Figure 7a), from approximately 2.5 to approximately 2.2 .

The level of GLCM entropy is continuously decreasing, and the most interesting fact regarding the evolution on levels is that levels $1+2$ and 3 are continuously decreasing, and 4+5 levels are increasing in the analyzed interval, which reveals that the turnover in this region are continuously growing, mostly in

DOI: $10.24818 / 18423264 / 54.1 .20 .04$ 
Daniel Peptenatu, Ion Andronache, Andreea Karina Gruia, Alexandra Grecu, Cristina Dima, Răzvan Cătălin Dobrea, Dumitru Alexandru Bodislav

the ATUs located in close proximity to Bucharest, and levels $1+2$ pass directly to $4+5$ considering that level 3 is also continuously decreasing.

The economic crisis is marked by a much lower growth of entropy in this region, compared to the others where it was much higher. Figure 7 cshows that during the economic crisis, $1+2$ levels fell to the detriment of level 3, indicating that many ATUs registering small turnover in this period increased to level 3 or $4+5$ because the latter are constantly growing, even during the crisis.

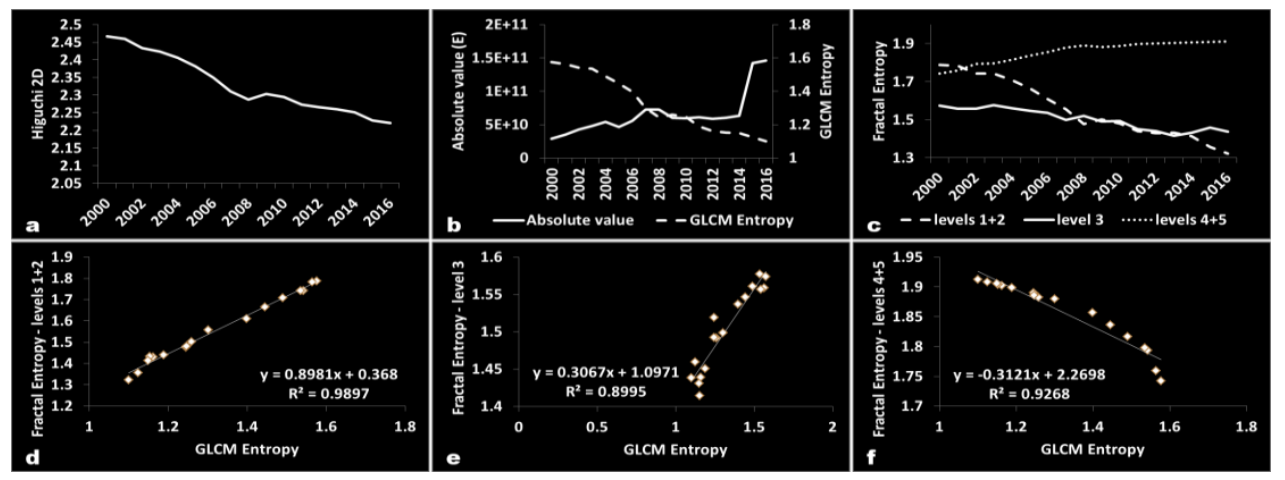

Figure 7. GLCM and fractal entropy dynamics-South Muntenia Development Region and Bucharest-Ilfov Development Region: a.- H2D dynamics; b.-GLCM entropy dynamics; c.-structural dynamics of fractal entropy; d.-correlation between GLCM entropy and the fractal entropy of low values levels (1+2); e.- correlation GLCM entropy and the fractal entropy of medium values-level 3; f.- correlation of GLCM entropy and the fractal entropy high value levels $(4+5)$.

Although the South-West Development Region Oltenia is the second poorest in Romania, it recorded the smallest decrease of entropy among all development regions. The enthalpy analysis of H2D and GLCM for this region shows the same decreasing trends as at national level, but these decreases are even lower than those of the North-East Development Region. As for the evolution of H2D (Figure 8a), it decreases from approximately 2.5 to 2.4 and GLCMentropy (Figure $8 \mathrm{~b}$ ) shows very low decreases from approximately 1.55 to 1.45 which is worrying even when compared to North East Development Region showing a greater decrease from approximately 1.55 to 1.3 .

Figure 8c shows an evolution in which levels $1+2$ decrease slightly but stay higher throughout the analyzed period than level 3, which also recorded slight increases especially after the economic crisis when it recorded slight decreases. Levels $4+5$ are in slow growth, maintaining relatively close to levels $1+2$.

The causes for which entropy in its evolution did not show major decrease in this region as recorded by other development regions may be multiple. These include the fact that the South-West Region does not present very strong cities 
Application of Fractal-structural Methods in the Analysis of the Spatial Distribution of Turnover in Romania

recording a high emerging area, besides the city of Craiova, and besides this we can also find the lack of infrastructure and accessibility in the northern and northwestern areas of the region.
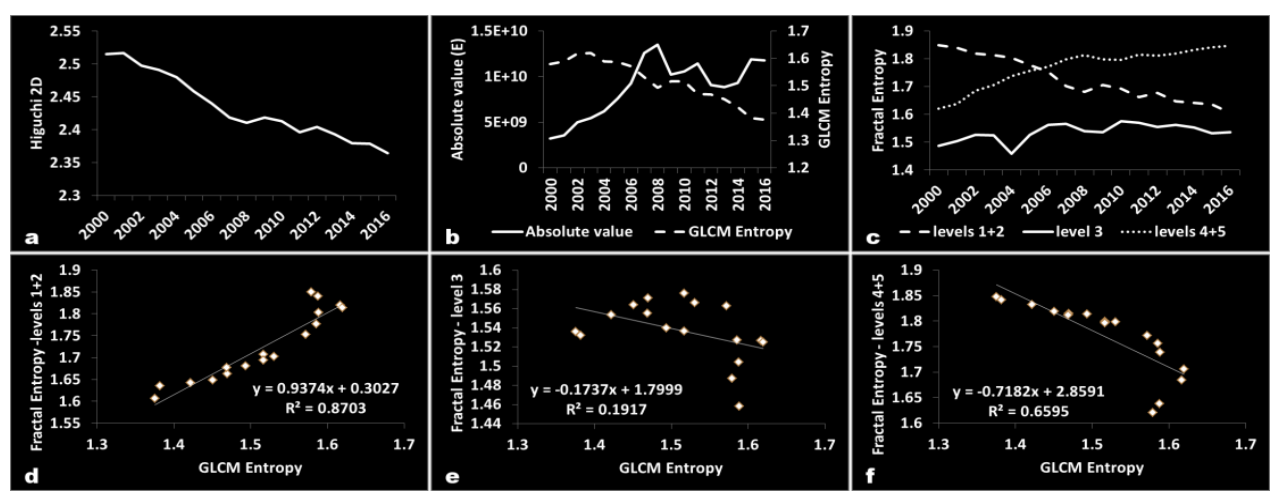

Figure 8. GLCM and fractal entropy dynamics-South-West Development Region Oltenia: a.- H2D dynamics; b.-GLCM entropy dynamics; c.-structural dynamics of fractal entropy; d.-correlation between GLCM entropy and the fractal entropy of low values levels $(1+2)$; e.- correlation GLCM entropy and the fractal entropy of medium values-level 3; f.- correlation of GLCM entropy and the fractal entropy high value levels $(4+5)$.

In Figure 9a we observe that even in the Western Region, the GLCM entropy has continuous decreasing tendencies, with the exception of the growth from the crisis period, growth much more significant than in the case of the Southern Region, which reveals that Timisoara is not able to exert the same polarizing power in the territory as the capital city, therefore the emerging area of this city is much smaller.

Turning to levels of turnover, levels $1+2$ decrease slightly over the entire period under review, registering a sharp decrease during the economic crisis, when a part of them went to level 3 that registered an increase even in this interval. Levels $4+5$ show an upward trend, this evolution being like the one in the Southern Region (Figure 9c).

Although entropy has been considerably reduced in the western part of the development region, in the eastern and southern parts, on the territories of the Caraș-Severin and Hunedoara counties, we still encounter a rather high degree of the fractal entropy, where levels $1+2$ and even 3 have an increased presence, compared to the rest of the region (Figures 9d, e, f).

DOI: $10.24818 / 18423264 / 54.1 .20 .04$ 
Daniel Peptenatu, Ion Andronache, Andreea Karina Gruia, Alexandra Grecu, Cristina Dima, Răzvan Cătălin Dobrea, Dumitru Alexandru Bodislav

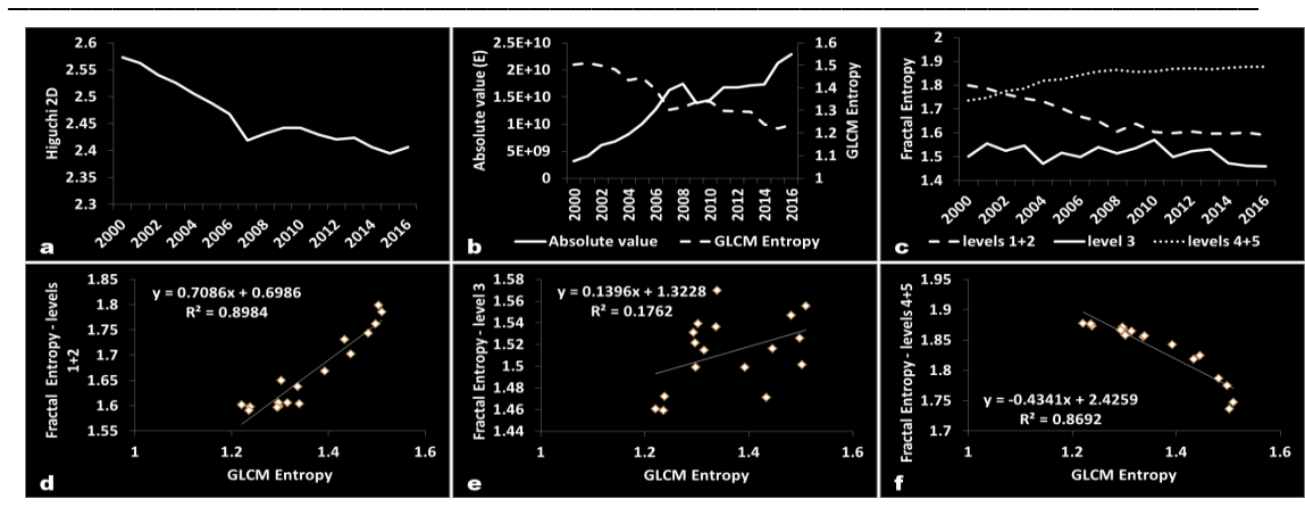

Figure 9. GLCM and fractal entropy dynamics-West Development Region: a.- H2D dynamics; b.-GLCM entropy dynamics; c.-structural dynamics of fractal entropy; d.-correlation between GLCM entropy and the fractal entropy of low values levels (1+2); e.- correlation GLCM entropy and the fractal entropy of medium values-level 3; f.- correlation of GLCM entropy and the fractal entropy high value levels $(4+5)$.

In the case of the North West Development Region, we note the polarizing role of Cluj-Napoca, which generates an emerging area around it, reducing entropy (Figure 10a, b). A small degree of entropy is recorded also in the western part of the region, where Oradea city succeeds to show its beneficial effects in the territory. The only regions where entropy is raised are those in the hills, namely Sălaj County, where Zalău and the other smaller towns in this area do not manage to attract investments and capital. GLCM entropy and H2D levels are also decreasing in this region, whereas levels $1+2$ show continuous decreases, while level 3 stagnating between 1.5 and 1.6, decreasing after 2011. Levels 4+5, as for the other regions, are slowly increasing continuously as a result of the turnover growth of several ATUs (Figures 10, c, d, e, f).

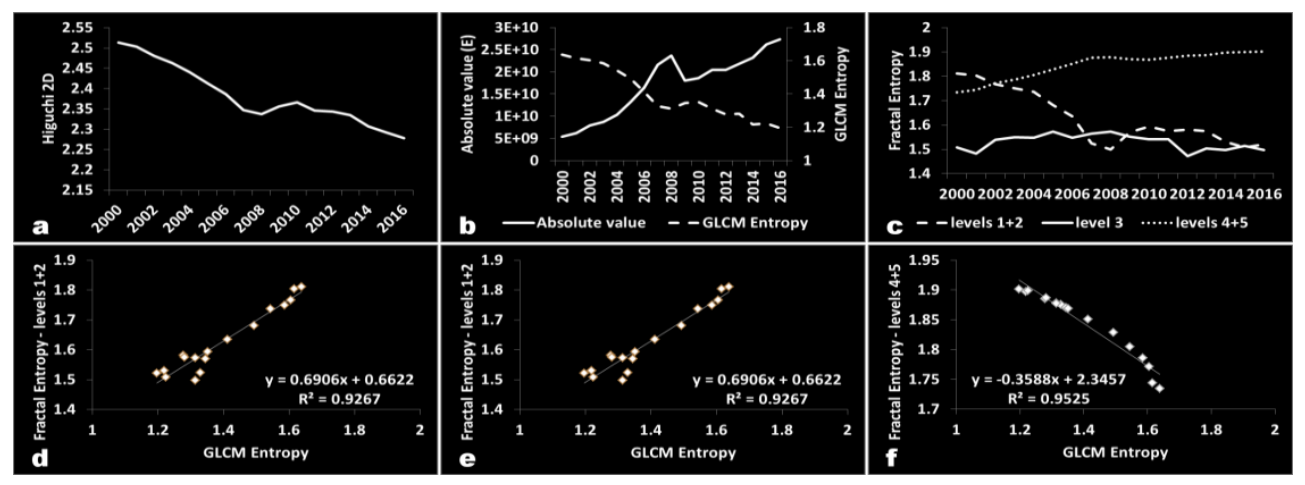

Figure 10. GLCM and fractal entropy dynamics-North-West Development 
Application of Fractal-structural Methods in the Analysis of the Spatial Distribution of Turnover in Romania

Region: a.- H2D dynamics; b.-GLCM entropy dynamics; c.-structural dynamics of fractal entropy; d.-correlation between GLCM entropy and the fractal entropy of low values levels $(1+2)$; e.- correlation GLCM entropy and the fractal entropy of medium values-level 3; f.- correlation of GLCM entropy and the fractal entropy high value levels $(4+5)$.

In the Central Development Region, the level of entropy is greatly reduced due to the economic growth of several cities in this area. Entropy is reduced primarily due to the main cities (Brasov, Sibiu, TârguMureş, Alba Iulia), which represent real poles of growth with a significant emerging area. A higher level of entropy is recorded in the center of the development region in the area adjacent to Medias, as it is not able to exert any major influence on a vast territory (Figure 11a, b).

The turnover levels are in line with the general national trend in that levels $1+2$ decrease to the point wherein they reach almost level 3. Level 3 is maintained at an average of 1.5 but with low upward fluctuations after the economic crisis. Levels 4+5 starting in 2000 from the same point as levels $1+2$ are continuously increasing until 2016 (Figures 11c, d, e, f).
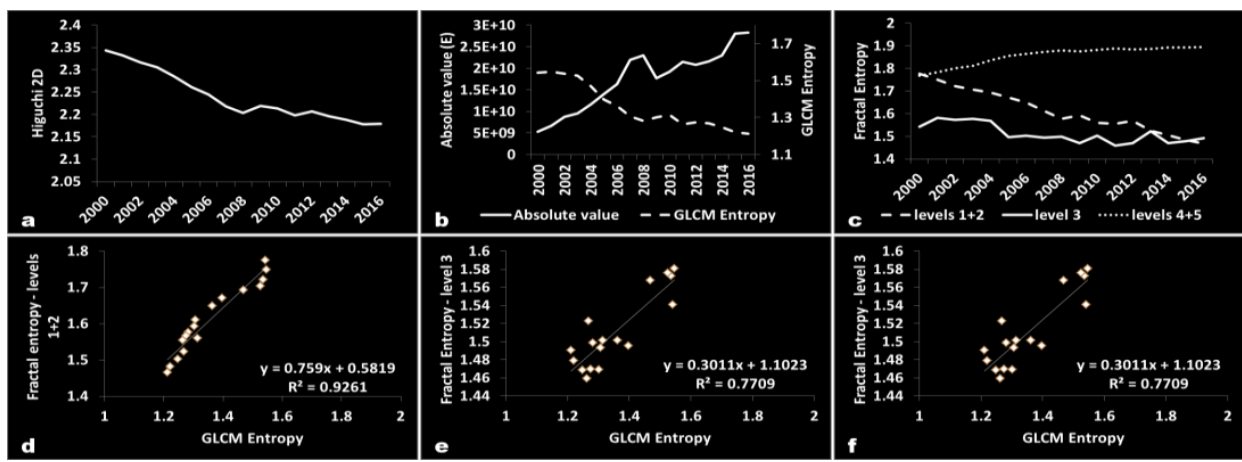

Figure 11. GLCM and fractal entropy dynamics-Center Development Region: a.- H2D dynamics; b.-GLCM entropy dynamics; c.-structural dynamics of fractal entropy; d.-correlation between GLCM entropy and the fractal entropy of low values levels $(1+2)$; e.- correlation GLCM entropy and the fractal entropy of medium values-level 3; f.- correlation of GLCM entropy and the fractal entropy high value levels $(4+5) .4$. Conclusions

The methods used in this study are meant to show the qualitative elements regarding the dynamics of the territorial profile, bringing a new dimension in research as compared to the classic economic and statistical methods which capture only the quantitative evolution of the economic dimension.

Through the evolution of GLCM entropy and of Higuchi2D analysis, grayscale images were generated that show the evolution of entropy and the complexity

DOI: 10.24818/18423264/54.1.20.04 
Daniel Peptenatu, Ion Andronache, Andreea Karina Gruia, Alexandra Grecu, Cristina Dima, Răzvan Cătălin Dobrea, Dumitru Alexandru Bodislav

in the territory between 2000 and 2016, with the general tendency to decrease. At national level, the decrease of entropy is given by the decrease of $1+2$ levels, maintaining level 3 at an average between 1.5 and 1.6 and a significant increase of levels $4+5$ that contribute massively to the reduction of entropy in the territory. The methodology used may contribute to increasing the relevance of analyzes based on the spatial dimension of the phenomenon analyzed (Albu et al. 2017; Pintilii et al. 2015;2016; 2017; Alexa et al. 2019).

Maintaining level 3 to a stable average over these years is because levels $1+2$ turn to 3 , and a part of level 3 pass in the upper levels $4+5$. Some of tiers 1 and 2 go directly to $4+5$, where ATUs are in the emerging area of big cities, without affecting level 3. Structural approach to development levels may be a methodological direction useful to substantiate the critical mass in manifestation of phenomena with spatial distribution (Pravălie et al. 2014; Lupu et al. 2016).

The highest increases of levels $4+5$ were recorded in urban systems with a population of over 250,000 inhabitants and in their emerging area, and hence dispersed to other areas, the capital city being the best example in this case. The lowest values, ranging from $1+2$, were recorded in the poorest areas, lacking infrastructure and investments at the ATU level, such as the North-East and SouthWest regions. Although entropy is considerably reduced within the analyzed period, in the last analyzed year, it still records a variable entropy degree, with significant levels of $1+2$ levels compared to the other development regions.

The development of methodologies for the analysis of processes and phenomena with spatial distribution will contribute to the consistent increase of the level of their understanding, of the awareness on the ways other components of the system contribute to the definition of the manifesting pattern.

\section{REFERENCES}

[1]Albu, L.L., Lupu, R., Calin, A. (2017), Risk Generating Industries for European Stock Markets. Economic Computation and Economic Cybernetics Studies and Research; ASE Publishing, 51, No. 4;

[2] Allen, T., Arkolakis, C. (2014), Trade and the Topography of the Spatial

Economy. The Quarterly Journal of Economics, 129 (3): 1085-1139;

[3] Alexa D., Cismas L.M., Rus A.V., Silaghi M.I.P. (2019), Economic Growth, Competitiveness and Convergence in the European Regions. A Spatial Model Estimation.Economic Computation and Economic Cybernetics Studies and Research; ASE Publishing, 1 (53): 107-124;

[4] Andronache, I., Fensholt, R., Ahammer, H., Ciobotaru, A.M., Pintilii, R.D., Peptenatu, D., Draghici, C.C., Diaconu, D.C., Radulovic, M., Pulighe, G., Azihou, A.F., Toyi, M.S., Sinsin, B. (2017), Assessment of Textural Differentiations in Forest Resources in Romania Using Fractal Analysis. Forests, 8 (3): 54; 
Application of Fractal-structural Methods in the Analysis of the Spatial Distribution of Turnover in Romania

[5] Boix, R., Trullén, J. (2007), Knowledge, Networks of Cities and Growth in Regional Urban Systems. Papers in Regional Science, 86 (4): 551-574;

[6] Cabrera, J.E. (2006), Texture Analyzer Plugin;

https://imagej.nih.gov/ij/plugins/texture.html;

[7] Di Ieva, A., Grizzi, F., Jelinek, H., Pellionisz, A.J., Losa, G.A. (2013),

Fractals in the Neurosciences, Part I: General Principles and Basic

Neurosciences. Neuroscientist, 20 (4): 403-417;

[8] Geppert, K., Stephan, A. (2008), Regional Disparities in the European

Union: Convergence and Agglomeration. Papers in Regional Science 87 (2): 193-

217;

[9]Haralick, R.M., Shanmugam, K., Dinstein, I. (1973), Texture Parameters for Image Classification. IEEE Transactions on Systems, Man and Cybernetics, 3 (6): 610-621;

[10] Harrison, A., McLaren, J., McMillan, M. (2011), Recent Perspectives on Trade and Inequality. Annual Review of Economics, 3: 261-289;

[11] Kainz, P., Mayrhofer-Reinhartshuber, M., Ahammer, H. (2015), IQM: An

Extensible and Portable Open Source Application for Image and Signal

Analysis.PLOS ONE, 10 (1): e0116329;

[12] Kunicki, A.C.B., Oliveira, A.J., Mendonça, M.B.M., Barbosa, C.T.F., Nogueira, R.A. (2009), Can the Fractal Dimension Be Applied for the Early

Diagnosis of Non-Proliferative Diabetic Retinopathy? Braz J. Med. Biol. Res. 42, 930-934;

[13] Lupu, I., Hurduzeu, G., Nicolae, M. (2016),Connections between Sentiment Indices and Reduced Volatilities of Sustainability Stock Market Indices. Economic Computation and Economic Cybernetics Studies and Research;ASE Publishing, 50(1);

[14] Meijers, E.J., Waterhout, B., Zonneveld, W.A.M. (2007), Closing the GAP: Territorial Cohesion through Polycentric Development. European Journal of Spatial Development, 24;

[15] Parker, E. (2017), The Entropic Linkage between Equity and Bond Market Dynamics .Entropy, 19(6): 292;

[16] Pintilii, R.D., Andronache, I., Diaconu, D.C., Dobrea, R.C., Zelenakova, M., Fensholt, R., Peptenatu, D., Draghici, C.C., Ciobotaru, A.M. (2017), Using Fractal Analysis in Modeling the Dynamics of Forest Areas and Economic Impact Assessment: Maramures County, Romania, as a Case Study. Forests, 8(1): 25 ;

[17]Pintilii, R. D., Andronache, I., Simion, A. G., Drăghici, C. C., Peptenatu D., Ciobotaru, A. M., Dobrea, R. C.,Papuc, R. M. (2016), Determining forest fund evolution by fractal analysis (Suceava-Romania), Urbanism Architecture Constructions, vol. 7, pp 31-42; 
Daniel Peptenatu, Ion Andronache, Andreea Karina Gruia, Alexandra Grecu, Cristina Dima, Răzvan Cătălin Dobrea, Dumitru Alexandru Bodislav

[18]Pintilii, R.D., Peptenatu, D., Draghici, C., Irina, S., Stoian, R.,D.(2015), Structural Changes in the Entrepreneurial Profile of the Creative Industries in Romania, 2nd Global Conference on Business, Economics, Management and Tourism, Edited by:Iacob, AI, Procedia Economics and Finance, Vol.23, pp:11471151.

[19] Pitsianis, N., Bleris, G., Argyrakis, P. (1989), Information Dimension in Fractal Structures. Physical Review B, 39(10): 7097;

[20] Pravalie, R., Sirodoev, I., Patriche, C.V., Bandoc, G., Peptenatu D. (2014), TheAnalysis of the Relationship between Climatic Water Deficit and Corn Agricultural Productivity in the Dobrogea Plateau. Carpathian Journal of Earth and Environmental Sciences, 9(4): 201-214;

[21] Schneider, C.A., Rasband, W.S., Eliceiri, K.W. (2012), NIH Image to Image J: 25 Years of Image Analysis. Nature methods, 9(7): 671-675;

[22] Spasić, S. (2014), On 2D Generalization of Higuchi's Fractal

Dimension.Chaos, Solitons \& Fractals, 69: 179-187. 